\title{
RINGS GRADED BY POLYCYCLIC-BY-FINITE GROUPS
}

\author{
WILLIAM CHIN AND DECLAN QUINN
}

(Communicated by Donald Passman)

\begin{abstract}
We use the duality between group gradings and group actions to study polycyclic-by-finite group-graded rings. We show that, for such rings, graded Noetherian implies Noetherian and relate the graded Krull dimension to the Krull dimension. In addition we find a bound on the length of chains of prime ideals not containing homogeneous elements when the grading group is nilpotent-by-finite. These results have suitable corollaries for strongly groupgraded rings. Our work extends several results on skew group rings, crossed products and group-graded rings.
\end{abstract}

Introduction. An associative ring with identity is said to be graded by the group $G$ if

$$
R=\bigoplus \sum_{x \in G} R(x)
$$

is a direct sum of additive subgroups $R(x)$ with $R(x) R(y) \subseteq R(x y)$. It follows that $1_{R} \in R(1) . R$ is said to be strongly $G$-graded if $R(x) R(y)=R(x y)$ for all $x, y \in G$.

The group $G$ is said to be polycyclic-by-finite if $G$ has a subnormal series $1=$ $G_{0} \triangleleft G_{1} \triangleleft \cdots \triangleleft G_{n}=G$, where $G_{i} / G_{i-1}$ is either infinite cyclic or finite. If $G$ has a normal series of this type, then $G$ is said to be strongly polycyclic-by-finite. The number of infinite cyclic factors which occur in this series is called the Hirsch number of $G$ and is denoted by $h(G)$. Since any two series have isomorphic refinements, $h(G)$ is a nonzero integer invariant of $G$.

In their paper [6] M. Cohen and S. Montgomery proved a duality theorem for finite group gradings and group actions on rings. Their methods provided a way to translate results on finite crossed products to more general group-graded rings. Their construction was developed concretely by the second author in [11], where further applications were given. In addition the approach there works for infinite groups. Here we use this method to study Krull dimension and chains of prime ideals in rings graded by polycyclic-by-finite groups. The construction of [11] for infinite groups is sketched in $\S 1$.

We comment that the duality theorem of Cohen and Montgomery has been extended by R. Blattner and S. Montgomery [4] to handle certain infinite groups as a corollary to a more general theorem on Hopf algebra actions. Other studies of the duality have been made by M. van den Bergh [2] and J. Osterburg [10].

We now describe the main results of this paper. Let $R$ be graded by a polycyclicby-finite group $G$. In $\S 2$ we show that a graded $R$-module $M$, which is graded Noetherian, is in fact Noetherian. Also in this situation, we show that the Krull

Received by the editors October 7, 1986.

1980 Mathematics Subject Classification (1985 Revision). Primary 16A03, 16A55, 16A33; Secondary 16A24. 
dimension is bounded above by the graded Krull dimension of $M$ plus the Hirsch number of $G$. This was proved by P. F. Smith for group rings [12] and for rings graded by strongly polycyclic-by-finite groups by Nastasescu [9].

In the third section we consider a smaller class of groups and obtain bounds on the lengths of chains of prime ideals containing no homogeneous elements, extending results of the first author $[\mathbf{5}, \S 3]$, the incomparability result for finite group-graded rings [6, Theorem 7.1], and an unpublished result of Bergman [3] concerning $\mathbf{Z}$ graded rings. If $G$ is finitely generated nilpotent-by-finite, we obtain the bound $2^{h(G)}-1$ on the length of such chains of primes. If $G$ is assumed to be finitely generated abelian-by-finite we get a sharper bound, namely $h(G)$. As a corollary we compute bounds on the prime rank (i.e. the classical Krull dimension) of $R$ in terms of the graded prime rank of $R$. As a final corollary we assume that $R$ is strongly graded and show that certain primes have different intersections with the identity component.

1. The matrix embedding. Let $R$ be graded by the group $G . M_{G}(R)$ denotes the set of row and column finite matrices over $R$, with the rows and columns indexed by the elements of $G . M_{G}^{*}(R)$ is the ideal consisting of those matrices with only finitely many nonzero entries. Note that if $G$ is finite, $M_{G}^{*}(R)=M_{G}(R)$.

$R$ may be embedded in $M_{G}(R)$ via the map sending $r$ to $\tilde{r} \in M_{G}(R)$, where $\tilde{r}_{x, y}=r\left(x y^{-1}\right)$, the $x y^{-1}$ component of $r$. $\tilde{R} \# G$ is defined to be subring of $M_{G}(R)$ generated by $\tilde{R}$ and $\{p(x) \mid x \in G\}$, where $p(x)=e(x, x)$ is the matrix unit. This construction is not a smash product in the usual sense unless the grading group $G$ is finite.

The group $G$ embeds in $M_{G}(R)$ as permutation matrices. Here $g \in G$ is sent to $\bar{g}=\sum_{x \in G} e(x, x g)$. Thus $\bar{G}=\{\bar{g} \mid g \in G\}$ is a subgroup of the group of units of $M_{G}(R)$.

LEMMA 1.1 [11, LEMMA 2.1]. Let $R$ be a $G$-graded ring with $G$ infinite. Then

$$
\tilde{R} \# G=\tilde{R} \oplus\left(\bigoplus \sum_{x \in G} \tilde{R} p(x)\right)
$$

is a free $\tilde{R}$-module with basis $\{I\} \cup\{p(x) \mid x \in G\}$. Furthermore,

$$
\sum_{x \in G} \tilde{R} p(x)=\sum_{x, y \in G} R\left(x y^{-1}\right) e(x, y)
$$

is an ideal of $\tilde{R} \# G$, which is essential both as a left ideal and as a right ideal.

We denote the set $\sum_{g \in G}(\tilde{R} \# G) \bar{g}=(\tilde{R} \# G) \bar{G}$ by $R\{G\}$, and let $(\tilde{R} \# G)^{G}$ denote the fixed ring.

LEMMA $1.2[11$, LEMMA 2.2]. Let $R$ be a $G$-graded ring with $G$ infinite. Then

(i) $\bar{g}^{-1}(\tilde{R} \# G) \bar{g}=\tilde{R} \# G$ for all $g \in G$ and $(\tilde{R} \# G)^{G}=\tilde{R}$.

(ii) $R\{G\}=\bigoplus \sum_{g \in G}(\tilde{R} \# G) \bar{g}$ is a direct sum of additive groups.

(iii) $R\{G\}$ is a skew group ring of $\bar{G}$ over $\tilde{R} \# G$. 
(iv) $R\{G\}=\left(\bigoplus \sum_{g \in G} \tilde{R} \bar{g}\right) \oplus M_{G}^{*}(R)$ as additive groups and $M_{G}^{*}(R)$ is an ideal of $R\{G\}$ which is essential both as a left ideal and as a right ideal of $R\{G\}$.

Next we note that the constructions for modules over finite group-graded rings given in $[11, \S 1]$, work equally well when $G$ is infinite. Here $\operatorname{Col}_{G}(M)$ denotes the set of infinite column matrices over $M$ with finitely many nonzero entries. Since the elements of $M_{G}(R)$ are both row and column finite, $\operatorname{Col}_{G} M$ is again a left $M_{G}(R)$-module and hence a left $R\{G\}$-module.

If $M$ is a graded $R$-module, we let $\tilde{M}=\left\{v \in \operatorname{Col}_{G} M \mid v_{x} \in M(x)\right\}$, where $v_{x}$ is the entry in the $x$-position of $v$. We now restate [11, Lemma 1.12] for arbitrary groups. Since the proofs are identical, we omit the proof here.

LEMMA 1.3. Let $R$ be a $G$-graded ring and let $M$ be a graded $R$-module. Then

$$
\operatorname{Col}_{G} M=\bigoplus \sum_{g \in G} \bar{g} \tilde{M}
$$

and each $\bar{g} \tilde{M}$ is an $(\tilde{R} \# G)$-submodule of $\operatorname{Col}_{G} M$. Thus $\operatorname{Col}_{G} M$ is the $R\{G\}$ module induced from the $(\tilde{R} \# G)$-module $\tilde{M}$.

2. Krull dimension. We begin by considering the Krull dimension of the $R\{G\}$-modules $\operatorname{Col}_{G} M$ and $\tilde{M}$. Since $M_{G}^{*}(R) \subseteq R\{G\}$, it is clear that $R\{G\}$ submodules of $\operatorname{Col}_{G} M$ are of the form $\operatorname{Col}_{G} N$, where $N \subseteq M$ is an $R$-submodule of $M$. (Note that if $G$ is finite, $M_{G}^{*}(R)=M_{G}(R)$.) Also if $M$ is a graded $R$-module, then $(\tilde{R} \# G)$-submodules of $\tilde{M}$ are of the form $\tilde{N}$, where $N \subset M$ is a graded submodule of $M$. This follows since $\left\{\alpha \in M_{G}^{*}(R) \mid \alpha_{x, y} \in R\left(x y^{-1}\right)\right\} \subseteq \tilde{R} \# G$.

The next lemma follows immediately from the above observations. We write gr $K$-dim for graded Krull dimension.

LEMMA 2.1. Let $R$ be a group-graded ring and $M$ an $R$-module with Krull dimension. Then

$$
K-\operatorname{dim}_{R} M=K-\operatorname{dim}_{R\{G\}} \operatorname{Col}_{G} M .
$$

Furthermore, if $M$ is a graded module, then

$$
\operatorname{gr} K-\operatorname{dim}_{R} M=K-\operatorname{dim}_{\tilde{R}} \# G \tilde{M} .
$$

Let $R * G$ be a skew group ring of a polycyclic-by-finite group $G$ over an arbitrary ring $R$. Also assume ${ }_{R} M$ is a Noetherian left $R$-module. Then

(A) $(R * G) \otimes{ }_{R} M$ is a left Noetherian $(R * G)$-module.

(B) (P. F. Smith [12])

$$
K-\operatorname{dim}_{R * G}(R * G) \otimes_{R} M \leq K-\operatorname{dim}_{R} M+h(G) .
$$

The statement in (A) is a well-known version of the Hilbert Basis Theorem. It is well known that Noetherian modules have Krull dimension (see [7]).

We can now prove the main result of this section.

THEOREM 2.2. Let $R$ be a $G$-graded ring where $G$ is a polycyclic-by-finite group. If $M$ is a graded Noetherian $R$-module then $M$ is Noetherian as an $R$ module. Furthemore $K-\operatorname{dim}_{R} M \leq \operatorname{gr} K-\operatorname{dim}_{R} M+h(G)$.

Proof. Since $M$ is graded Noetherian, $\tilde{M}$ is Noetherian as an $(\tilde{R} \# G)$-module. Thus we can use Lemma 1.3 and (A) applied to $\tilde{M}$ to conclude that $\operatorname{Col}_{G} M$ is 
Noetherian as an $R\{G\}$-module. It now follows that $M$ is Noetherian as an $R$ module.

We can also apply (B) to deduce that $K-\operatorname{dim}_{R\{G\}} \operatorname{Col}_{G} M \leq K-\operatorname{dim}_{\tilde{R} \# G} \tilde{M}+$ $h(G)$. In view of Lemma 2.1 , this implies that $K-\operatorname{dim}_{R} M \leq \operatorname{gr} K-\operatorname{dim}_{R} M+$ $h(G)$.

If $R$ is strongly $G$-graded, then graded left ideals of $R$ are generated by their intersection with $R(1)$. Thus $R$ is graded Noetherian if and only if $R(1)$ is Noetherian. Also if $K-\operatorname{dim} R(1)$ exists, then $g r K-\operatorname{dim} R$ exists and equals $K-\operatorname{dim} R(1)$.

The first assertion of the following corollary was proved by A. Bell $[1$, Proposition $2.5]$.

COROLLARY 2.3. Let $R$ be strongly graded by the polycyclic-by-finite group $G$. Then $R$ is left Noetherian if and only if $R(1)$ is left Noetherian. Furthermore, if $R(1)$ is left Noetherian, then $K-\operatorname{dim} R \leq K-\operatorname{dim} R(1)+h(G)$.

ProOF. From the discussion above, $R$ is graded left Noetherian if and only if $R(1)$ is left Noetherian. But from Theorem 2.2, $R$ is left graded Noetherian precisely when $R$ is left Noetherian.

Now if $R(1)$ is left Noetherian, then by Theorem 2.2 again,

$$
K-\operatorname{dim} R \leq \operatorname{gr} K-\operatorname{dim} R+h(G)=K-\operatorname{dim} R(1)+h(G)
$$

We conclude this section with an example to show that when $R$ is not strongly graded $K-\operatorname{dim} R(1)$ and $\operatorname{gr} K-\operatorname{dim} R$ need not be related, even if $G=\mathbf{Z}$.

EXAMPLE 2.4. Let $R=F\left[X_{1}, \ldots, X_{n}\right]$ where $F$ is a field. We can grade $R$ by total degree so that if $k \geq 0, R(k)$ is spanned over $F$ by $\left\{X_{1}^{m_{1}} \cdots X_{n}^{m_{n}} \mid m_{1}+\right.$ $\left.\cdots+m_{n}=k\right\}$ and $R(k)=0$ if $k<0$. Thus $R$ is Z-graded and Noetherian. $R(0)=F$, so that $K-\operatorname{dim} R(0)=0$. But $\operatorname{gr} K-\operatorname{dim} R=n$. Indeed, it is clear that gr $K-\operatorname{dim} R \leq K-\operatorname{dim} R=n$. The reverse inequality follows by induction from the observation that $R \supseteq R X_{1} \supseteq R X_{1}^{2} \supseteq \cdots$ is a decreasing sequence of graded ideals and

$$
\frac{R X_{1}^{i}}{R X_{1}^{i-1}} \cong F\left[X_{2}, \ldots, X_{n}\right] .
$$

3. Chains of prime ideals. The main purpose of this section is to obtain a bound on the lengths of chains of prime ideals of a $G$-graded ring $R$ which contain no homogeneous elements.

An element $r \in R$ is said to be homogeneous if $0 \neq r \in R(x)$ for some $x \in G$. A group $G$ is said to be nilpotent-by-finite if $G$ has a nilpotent normal subgroup of finite index. Abelian by finite groups are defined analogously. In this section we assume that $G$ is, at least, finitely generated nilpotent-by-finite. It is elementary that these groups are polycyclic-by-finite.

In [6] Cohen and Montgomery generalized incomparability for crossed products of finite groups [8]. What is done here is to prove analogous results, namely Theorem 3.2 and its corollaries, for rings graded by certain possibly infinite groups.

Let us begin by stating a minor extension of [5, Theorems 3.9 and 3.10].

The proof is identical if one observes that a finitely generated nilpotent-by-finite group is, in fact, torsion-free nilpotent-by-finite. 
THEOREM 3.1. Let $P_{0} \varsubsetneqq P_{1} \varsubsetneqq \ldots \varsubsetneqq P_{n}$ be a chain of prime ideals of a crossed product $R * G$. If either

(i) $G$ is finitely generated nilpotent-by-finite with $n \geq 2^{h(G)}$, or

(ii) $G$ is finitely generated abelian-by-finite with $n>h(G)$, then $P_{0} \cap R \neq P_{n} \cap R$.

Next we state the graded version of this result.

THEOREM 3.2. Let $R$ be a ring graded by a group $G$. If $P_{0} \varsubsetneqq \ldots \varsubsetneqq P_{n}$ is a chain of prime ideals of $R$, and $G$ satisfies (i) or (ii) in the theorem above, then $P_{n}$ contains a homogeneous element.

This theorem was proved by G. Bergman [3] in the case where $G$ is infinite cyclic by means of a direct computational proof.

Before proceeding with the proof of Theorem 3.2 it is useful to define the following ideal maps, whose basic properties are described in the subsequent three lemmas.

DEFINITION (i) For an ideal $I$ of $R$ define

$$
I^{u}=M_{G}(I) \cap R\{G\} .
$$

(ii) For an ideal $J$ of $R\{G\}$ define

$$
J^{d}=J_{1,1}=\left\{\alpha_{1,1} \mid \alpha \in J\right\} .
$$

It follows easily that $I^{u}$ is an ideal of $R\{G\}$ and $J^{d}$ is an ideal of $R$. Also observe that $M_{G}^{*}(I) \subset I^{u}$, and the maps ${ }^{u}$ and ${ }^{d}$ preserve inclusions.

LEMMA 3.3. Let $I$ be an ideal of $R$. Then $I^{u d}=I$.

ProOF. Let $a \in I$. Since $a e(1,1) \in M_{G}^{*}(I) \subset I^{u}$ we have $a \in I^{u d}$. Conversely, if $a \in I^{u d}$, there exists $\alpha \in I^{u}$ with $\alpha_{11}=a$. Since $I^{u} \subset M_{G}(I)$, we obtain $a \in I$.

LEMMA 3.4. Let $J, K$ be ideals of $R\{G\}$. Then $J^{d} \cdot K^{d} \subset(J K)^{d}$.

ProOF. Note that $J^{d} e(1,1) \subset J$ since $e(1,1) \in R\{G\}$. With this the result is immediate from the definition.

LEMMA 3.5. If $P$ is a prime ideal of $R$, then $P^{u}$ is a prime ideal of $R\{G\}$.

ProOF. Suppose $J K \subset P^{u}$ where $J, K$ are ideals of $R\{G\}$. Using the previous two lemmas, we set that

$$
J^{d} K^{d} \subset(J K)^{d} \subset P^{u d}=P .
$$

Thus $J^{d}$ or $K^{d}$ is contained in $P$, say $J^{d}$.

We show that $J \subset P^{u}$. Let $\alpha \in J$, and $x, y \in G$. Set

$$
\beta=e(1, x) \alpha e(y, 1)=\alpha_{x, y} e(1,1) .
$$

Notice that $\beta \in J$ because $J$ is an ideal and $R\{G\} \supseteq M_{G}^{*}(R)$. Thus $\beta_{11}=\alpha_{x, y} \in$ $J^{d} \subset P$. We have shown that $\alpha=\left(\alpha_{x, y}\right) \in M_{G}(P)$; thus $\alpha \in M_{G}(P) \cap J \subset$ $M_{G}(P) \cap R\{G\}=P^{u}$. Hence $J \subset P^{u}$, showing that $P^{m}$ is prime.

PROOF OF THEOREM 3.2. Let $P_{1} \varsubsetneqq \ldots \varsubsetneqq P_{n}$ be a chain of primes of $R$. Applying Lemma 3.5, we obtain

$$
P_{0}^{u} \varsubsetneqq \cdots \varsubsetneqq P_{n}^{u}
$$


a chain of primes of $R\{G\}$, where the containments are strict by Lemma 3.3. Using Lemma $1.2 R\{G\}$ is a skew group $\operatorname{ring}(\tilde{R} \# G) \bar{G}$ with $\bar{G} \cong G$. Now Theorem 3.1 yields $P_{n}^{u} \cap(\tilde{R} \# G) \neq 0$.

Let $\alpha$ be a nonzero element of $P_{n}^{u} \cap(\tilde{R} \# G)$. Then observe that the entries of $\alpha$ are in $P_{n}$ since $P_{n}^{u} \subset M_{G}\left(P_{n}\right)$ and, furthermore, $\alpha_{x, y} \in R\left(x y^{-1}\right)$ for all $x, y \in G$, using Lemma 1.1. Finally, since $\alpha \neq 0$,

$$
0 \neq \alpha_{x, y} \in P_{n} \cap R\left(x y^{-1}\right)
$$

for some $x, y \in G$, so $P_{n}$ contains a homogeneous element.

A graded ideal $P$ is said to be graded prime if the product of graded ideals, which are not contained in $P$, cannot be contained in $P$. If $I$ is any ideal, let $I_{G}$ denote the largest graded ideal contained in $I$. If $I$ is a prime ideal, it follows that the contraction $I_{G}$ is graded prime.

Naturally, one may examine the prime rank of $R$ (classical Krull dimension) in terms of the graded prime rank of $R$. In particular, Theorem 3.2 says that, by factoring out $P_{0 G}$, a chain of at most $h(G)+1$ primes can contract to a given graded prime if $G$ is abelian-by-finite, and at most $2^{h(G)}$ primes if $G$ is nilpotent-by-finite. As a consequence we obtain the following corollary.

COROLLARY 3.6. Let $R$ be as in Theorem 3.2, and let d denote the graded prime rank of $R$. Then the prime rank of $R$ is at most:

(i) $(h(G)+1)(d+1)-1$ if $G$ is abelian-by-finite

(ii) $2^{h(G)}(d+1)-1$ if $G$ is nilpotent-by-finite.

If we assume that $R$ is strongly graded by $G$, we can extend [6, Theorem 7.1].

COROLLARY 3.7. In addition to the hypotheses of Theorem 3.2, assume that $R$ is strongly graded by $G$. Then $P_{0} \cap R(1) \neq P_{n} \cap R(1)$.

Proof. Consider the factor ring $\bar{R}=R /\left(P_{0}\right)_{G}$. Then $\bar{R}$ is again strongly $G$-graded. Thus we may assume that $P_{0} \cap R(1)=0$. Theorem 3.2 now says that $P_{n} \cap R(x) \neq 0$ for some $x \in G$. Since $1 \in R\left(x^{-1}\right) R(x)=R(1)$, we have $\left(P_{n} \cap R(x)\right) R\left(x^{-1}\right) \neq 0$. Thus we conclude that

$$
0 \neq\left(P_{n} \cap R(x)\right) R\left(x^{-1}\right) \subset P_{n} \cap R(1) .
$$

ACKnowledgment. The authors would like to thank D. S. Passman for his helpful comments.

\section{REFERENCES}

1. A. D. Bell, Localization and ideal theory in Noetherian strongly group-graded rings, J. Algebra 105 (1987), 76-115.

2. M. Van den Bergh, A duality theorem for Hopf module algebras, Methods in Ring Theory, NATO ASI Series, vol. 129, Reidel, Dordrecht, 1983.

3. G. Bergman, Homogeneous elements and prime ideals in $\mathbf{Z}$-graded rings, preprint.

4. R. J. Blattner and S. Montgomery, A duality theorem for Hopf module algebras, J. Algebra 94 (1985), 153-172.

5. W. Chin, Prime ideals in differential operator rings and crossed products of infinite groups, J. Algebra 106 (1987), 78-104

6. M. Cohen and S. Montgomery, Group-graded rings, smash products and group actions, Trans. Amer. Math. Soc. 282 (1984), 237-258. 
7. R. Gordon and J. C. Robson, Krull dimension, Mem. Amer. Math. Soc. No. 133 (1973).

8. M. Lorenz and D. S. Passman, Prime ideals in crossed products of finite groups, Israel J. Math. 33 (1979), 89-132.

9. C. Nastasescu, Group rings of graded rings, applications, J. Pure Appl. Algebra 33 (1984), 315-335.

10. J. Osterburg, Smash products and G-Galois actions, Proc. Amer. Math. Soc. 98 (1986), 217221.

11. D. Quinn, Group-graded rings and duality, Trans. Amer. Math. Soc. 292 (1985), 154-167.

12. P. F. Smith, Corrigendum to "On the dimension of group rings", Proc. London Math. Soc. 3 (1973), 766-768.

Department of Mathematics, The University of Texas at Austin, Austin, TEXAS 78712

Department of MAthematics, University of Utah, SAlt LAKe City, Utah 84112 